\title{
Let's Do the Time Warp: Multicomponent Nonlinear Motion Estimation in Differential SAR Tomography
}

\author{
Xiao Xiang Zhu, Student Member, IEEE, and Richard Bamler, Fellow, IEEE
}

\begin{abstract}
In the differential synthetic aperture radar tomography (D-TomoSAR) system model, the motion history appears as a phase term. In the case of nonlinear motion, this phase term is no longer linear and, hence, cannot be retrieved by spectral estimation. We propose the "time warp" method that rearranges the acquisition dates such that a linear motion is pretended. The multicomponent generalization of time warp rewrites the D-TomoSAR system model to an $(M+1)$-dimensional standard spectral estimation problem, where $M$ indicates the user-defined motion model order and, hence, enables the motion estimation for all possible complex motion models. Both simulations and real data (from TerraSAR-X spotlight) examples demonstrate the applicability of the method and show that linear and periodic (seasonal) motion components can be separated and retrieved.
\end{abstract}

Index Terms-Differential synthetic aperture radar tomography (D-TomoSAR), multicomponent nonlinear motion, TerraSAR-X (TS-X), synthetic aperture radar (SAR), time warp.

\section{INTRODUCTION}

D IFFERENTIAL synthetic aperture radar tomography (D-TomoSAR) uses multibaseline multitemporal SAR acquisitions for reconstructing the 3-D distribution of scatterers and their motion [1]-[3]. For each azimuth-range $(x-r)$ pixel, a scattering profile along the third dimension, elevation $(s)$, is estimated. Ideally, a motion history (e.g., subsidence or building deformation) can be assigned to each elevation position. In many cases, e.g., in urban environment, these scattering profiles are dominated by a few individual scatterers only, typically, zero, one, or two.

The elevation estimation of a scatterer exploits the linear relationship between the measured phase and the product of baseline and elevation, and hence, the full arsenal of spectral estimation methods can be applied. Motion, however, is often nonlinear (periodic, accelerating, stepwise, etc.) and, hence, does not fit well into the spectral analysis framework. This

Manuscript received October 25, 2010; revised November 29, 2010; accepted December 23, 2010. This work was supported by the International Graduate School of Science and Engineering, Technische Universität München, München.

X. Zhu is with Lehrstuhl für Methodik der Fernerkundung, Technische Universität München, 80333 München, Germany (e-mail: xiaoxiang.zhu@bv. tum.de).

R. Bamler is with the Remote Sensing Technology Institute (IMF), German Aerospace Center (DLR), 82234 Wessling, Germany, and also with the Lehrstuhl für Methodik der Fernerkundung, Technische Universität München, 80333 München, Germany (e-mail: richard.bamler@dlr.de).

Color versions of one or more of the figures in this paper are available online at http://ieeexplore.ieee.org.

Digital Object Identifier 10.1109/LGRS.2010.2103298 is particularly true if very high-resolution mapping of urban infrastructure is to be performed. In this letter, we introduce nonlinear warping of the time axis to accommodate nonlinear motion models. The method is extended to multicomponent motion.

\section{IMAGING MODEL}

\section{A. General $(3+M)$-Dimensional SAR Imaging Model}

The focused complex-valued measurement $g_{n}$ at an azimuth-range pixel for the $n$th acquisition at time $t_{n}(n=$ $1, \ldots, N)$ is [2]

$$
g_{n}=\int_{\Delta s} \gamma(s) \exp \left(-j 2 \pi\left(\xi_{n} s+2 d\left(s, t_{n}\right) / \lambda\right)\right) d s
$$

where $\gamma(s)$ represents the reflectivity function along elevation $s$ with an extent of $\Delta s$ and $\xi_{n}=-2 b_{n} /(\lambda r)$ is the spatial (elevation) frequency proportional to the respective aperture position (baseline) $b_{n} \cdot d\left(s, t_{n}\right)$ is the line-of-sight (LOS) motion as a function of elevation and time. The motion relative to the master acquisition may be modeled using a linear combination of $M$ base functions $\tau_{m}\left(t_{n}\right)$

$$
d\left(s, t_{n}\right)=\sum_{m=1}^{M} p_{m}(s) \tau_{m}\left(t_{n}\right)
$$

where $p_{m}(s)$ is the corresponding motion coefficient to be estimated. In Section III, we will show that $\tau_{m}\left(t_{n}\right)$ can be interpreted as a warped time variable if we choose the units of the coefficients appropriately. The choice of the base functions depends on the underlying physical motion processes.

\section{B. Special Case I: PSI System Model}

Persistent scatterer (PS) interferometry (PSI) assumes the presence of only a single dominant scatterer in each pixel, and only the phase of each acquisition is used

$$
\arg \left(g_{n}\right)=2 \pi\left(\xi_{n} s_{0}+2 d\left(t_{n}\right) / \lambda\right)
$$

This restriction means that the motion depends only on $t_{n}$, i.e., $d\left(t_{n}\right)=\sum_{m=1}^{M} p_{m} \tau_{m}\left(t_{n}\right)$, and the motion parameters and the elevation of each scatterer can be estimated by model fitting and phase unwrapping, e.g., by the LAMBDA algorithm [4]. 


\section{Special Case II: D-TomoSAR With Linear Motion}

By assuming a linear motion with (elevation-dependent) velocity $V(s)$ (but possibly several scatterers per pixel), (1) simplifies to

$$
g_{n}=\int_{\Delta s} \gamma(s) \exp \left(-j 2 \pi\left(\xi_{n} s+\eta_{n} V(s)\right)\right) d s
$$

where $\eta_{n}=2 t_{n} / \lambda$ is the so-called "velocity frequency." Equation (4) can be rewritten as [5]

$$
\begin{array}{r}
g_{n}=\iint_{\Delta s} \gamma(s) \delta(v-V(s)) \exp \left(-j 2 \pi\left(\xi_{n} s+\eta_{n} v\right)\right) d v d s, \\
n=1, \ldots, N
\end{array}
$$

where $\Delta v$ is the range of possible velocities. Equation (5) is a 2-D Fourier transform of $\gamma(s) \delta(v-V(s))$ which is a delta line in the elevation-velocity $(s-v)$ plane along $v=V(s)$. Its projection onto the elevation axis $\gamma(s) \delta(v-V(s))$ is the reflectivity profile $\gamma(s)$.

If only $K$ discrete scatterers are present, $\gamma(s) \delta(v-V(s))$ consists of $K \delta$-points in the 2-D $(s-v)$ plane at the respective elevations and velocities. The tomographic reconstruction will be a blurred and noisy version of these peaks from which elevations and velocities are estimated.

\section{Velocity Spectrum}

In the original D-TomoSAR paper [2], the concept of the velocity spectrum has been introduced. It generalizes the system model (5) by replacing $\delta(v-V(s))$ by an arbitrary motion term $a(s, v)$

$$
g_{n}=\int_{\Delta s} \int_{\Delta v} \gamma(s) a(s, v) \exp \left(-j 2 \pi\left(\xi_{n} s+\eta_{n} v\right)\right) d v d s
$$

where

$$
\int_{\Delta v} a(s, v) \exp \left(-j 2 \pi \eta_{n} v\right) d v=\exp \left(-j \frac{4 \pi}{\lambda} d\left(s, t_{n}\right)\right) .
$$

Estimates of $\gamma(s) a(s, v)$ are derived from the measurements $g_{n}$ by 2-D spectral analysis. Individual scatterers with linear motion will show up as peaks in the $(s-v)$ plane at their respective elevations and velocities. In the case of moderately nonlinear motion and sufficient SNR, these peaks are blurred in the $v$-direction, but their elevations are still detectable [2]. Then, for each of these elevations, a Fourier transform in the $v$-direction leads to an estimate of $\exp \left(-j 4 \pi d\left(s, t_{n}\right) / \lambda\right)$ and, hence, of the motion $d\left(s, t_{n}\right)$ [2], [6].

This method works well with single scatterers and for multiple scatterers as long as they can be separated in the $(s, v)$ plane. There are, however, situations where it fails.

1) If the motion is too nonlinear, e.g., periodic, and the signal-to-noise ratio (SNR) is not high enough, the scatterer cannot be detected in the $(s, v)$ plane.

2) If two scatterers are closer in elevation and mean velocity than the respective resolution elements, their velocity spectra will influence each other with an unpredictable effect on the reconstruction of $d\left(s, t_{n}\right)$.

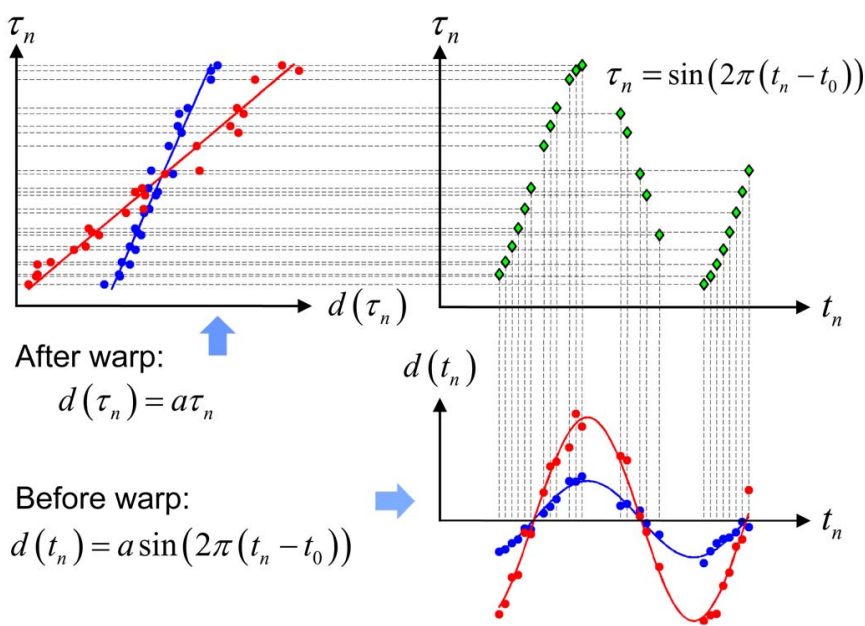

Fig. 1. Time warp converts seasonal periodic motions with different amplitudes (red and blue) into linear functions of different slopes.

From this discussion, it is evident that we need a transformation that is able to generate peaks of high SNR in some motion coefficient space just like spectral estimation works for linear motion.

\section{Generalized Time Warp Method}

We have briefly introduced an approach in [7] to cope with the problem of D-TomoSAR reconstruction with singlecomponent nonlinear motion by rearranging the acquisitions on the time axis (the so-called "time warp"). In this letter, we generalize it to the multicomponent model.

\section{A. Time Warp for $M=1$}

In the case of $M=1$, by introducing the temporal frequency $\eta_{1, n}=2 \tau_{1, n}\left(t_{n}\right) / \lambda$ as a function of a motion base function $\tau_{1, n}\left(t_{n}\right)$, and the motion coefficient $p_{1}(s)$, the proposed time warp method leads to a generalized system description which can be adapted for different nonlinear motion models

$$
\begin{array}{r}
g_{n}=\iint_{\Delta p \downarrow s} \gamma(s) \delta\left(p_{1}-p_{1}(s)\right) \exp \left(-j 2 \pi\left(\xi_{n} s+\eta_{1, n} p_{1}\right)\right) d s d p_{1}, \\
n=1, \ldots, N .
\end{array}
$$

The time warp rewrites the D-TomoSAR model with singlecomponent motion (linear or nonlinear) to a standard 2-D spectral estimation problem which makes all spectral estimators applicable. This principle is shown for a periodic motion model in Fig. 1. The most common motion base functions are as follows.

1) Linear motion: $\eta_{1, n}=2 t_{n} / \lambda$, and the coefficient $p_{1}(s)$ stands for the LOS velocity $(v)$ as a function of $s$.

2) Seasonal motion: $\tau_{1}\left(t_{n}\right)=\sin \left(2 \pi\left(t_{n}-t_{0}\right)\right)$, and $p_{1}(s)$ stands for the amplitude $(a)$ of the periodic motion; $t_{0}$ is the initial phase offset.

3) Thermal dilation: $\tau_{1}\left(t_{n}\right)=T\left(t_{n}\right)$, i.e., the temperature, and $p_{1}(s)$ is the scaling factor of the thermal dilation along $s$. 

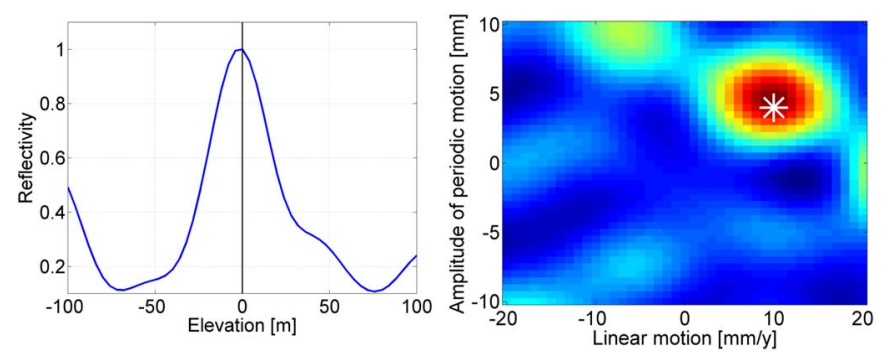

Fig. 2. Multicomponent motion reconstruction example with linear and seasonal periodic motion. Single scatterer with an elevation of $0 \mathrm{~m}$, a linear motion of $10 \mathrm{~mm} / \mathrm{year}$, and a seasonal motion with an amplitude of $4 \mathrm{~mm}$ $(\mathrm{SNR}=3 \mathrm{~dB})$. (Left) Projection of the 3-D spectral estimates to elevation direction (black line shows the true elevation position). (Right) Projection to the $a-v$ motion plane (asterisk marks the true position).
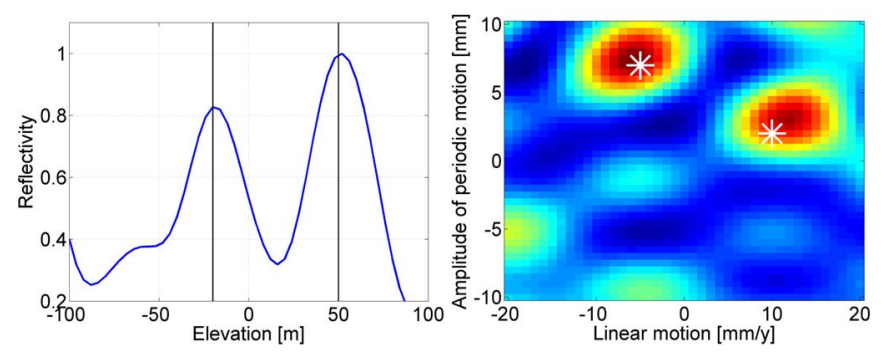

Fig. 3. Similar simulation as in Fig. 2 but with two scatterers inside the cell with elevations of $-20 / 50 \mathrm{~m}$, linear motion of $10 /-5 \mathrm{~mm} / \mathrm{year}$, and seasonal motion amplitudes of $2 / 7 \mathrm{~mm}$.

\section{B. Let's Do the Time Warp Again}

The generalization of the time warp method for $M>1$ is straightforward. Let us define the $m$ th temporal frequency component at $t_{n}$ as $\eta_{m, n}=2 \tau_{m}\left(t_{n}\right) / \lambda$.

Then, (1) can be rewritten as an $M+1$-dimensional Fourier transform of $\gamma(s) \delta\left(p_{1}-p_{1}(s), \ldots, p_{M}-p_{M}(s)\right)$ which is a delta line in the $M+1$ elevation-motion parameter space

$$
\begin{aligned}
& g_{n}=\int_{\Delta p_{M}} \ldots \int_{\Delta p_{1}} \int_{\Delta s} \gamma(s) \delta\left(p_{1}-p_{1}(s), \ldots, p_{M}-p_{M}(s)\right) \\
& \cdot \exp \left(-j 2 \pi\left(\xi_{n} s+\eta_{1, n} p_{1}+\cdots\right.\right. \\
&\left.\left.+\eta_{M, n} p_{M}\right)\right) \\
& \times d s d p_{1} \ldots d p_{M}, \quad n=1, \ldots, N
\end{aligned}
$$

This extension is a general solution to the multicomponent nonlinear motion estimation problem for D-TomoSAR and, hence, completes the time warp concept for complex motion models.

Multicomponent motion reconstruction examples, i.e., combined linear and seasonal motion, with single (see Fig. 2) and double scatterers (see Fig. 3) inside a resolution cell, respectively, are presented here to illustrate this concept. A realistic SNR of $3 \mathrm{~dB}$ and a baseline configuration of the TS-X "asterisk" stack with 30 acquisitions used in Section IV (see Fig. 5) are simulated in both examples. We start with a single scatterer located at zero elevation undergoing a linear motion of $10 \mathrm{~mm} / \mathrm{year}$ and a seasonal motion whose amplitude is $4 \mathrm{~mm}$. In this case, $M=2$; therefore, we are dealing with a 3-D spectral estimation problem. The left plot in Fig. 2 shows a projection of the 3-D spectral estimates to the elevation direction, i.e., a sum over the linear and periodic motion

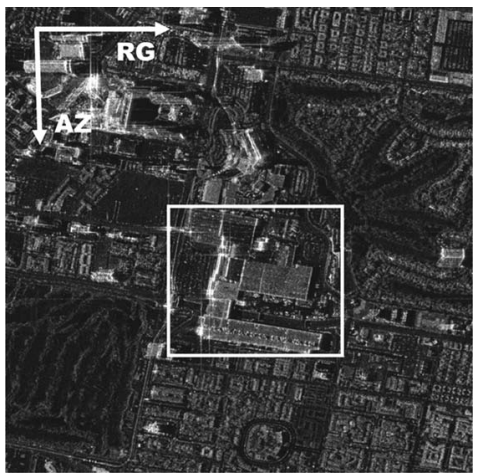

Fig. 4. Mean intensity map of the TS-X spotlight data stack over the test site in Las Vegas, Nevada. White box indicates the investigation area in Fig. 8.

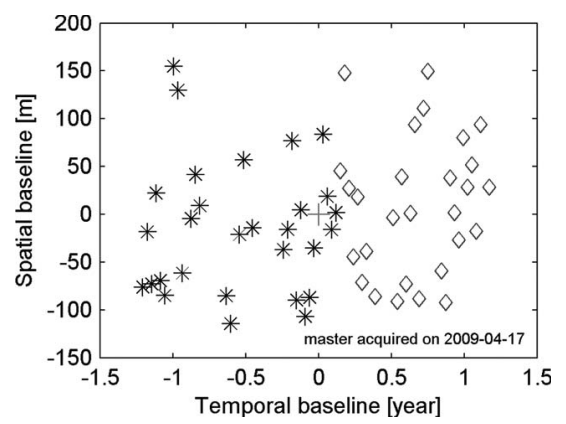

Fig. 5. Spatial-temporal baseline distribution. (Asterisks) Substack with seasonal motion only and (diamonds) substack with seasonal and linear motion.

plane. The right plot shows the corresponding projection in the amplitude-velocity $(a-v)$ plane, and the white asterisk indicates the true amplitude and velocity. Fig. 3 uses the same configuration as Fig. 2 but with two scatterers, located at -20 and $50 \mathrm{~m}$ (ca. $2 \times$ Rayleigh elevation resolution), with a linear motion of 10 and $-5 \mathrm{~mm} / \mathrm{year}$ and a seasonal motion with amplitudes of 2 and $7 \mathrm{~mm}$, respectively. Comparing the estimates to the simulation truth, it demonstrates that the generalized time warp method can give a robust estimation of the multicomponent nonlinear motion even under a moderate SNR.

\section{EXPERIMENTS With REAL DATA}

\section{A. Test Site}

The proposed method has been applied to TerraSAR-X highresolution spotlight data with a slant-range resolution of $0.6 \mathrm{~m}$ and an azimuth resolution of $1.1 \mathrm{~m}$, over the city of Las Vegas. A stack of 60 SAR images covering a time period of more than two years, from February 2008 to June 2010, is used. The preprocessing, including atmospheric phase screen correction, is performed by the German Aerospace Center's PSI-GENESIS system on a sparse PS network of high-SNR pixels containing only single scatterers. Fig. 4 shows a temporally averaged intensity map of the whole stack over the test area centered at the convention center in Las Vegas. Fig. 5 shows the elevation aperture positions and temporal baselines relative to the master image acquired on April 17, 2009.

This stack is particularly interesting for our purpose: No long-term motion has been observed in the test area over the first year of acquisition (the "asterisk" substack in Fig. 5), i.e., the motion-induced phase is only contributed by thermal dilation. Since July 2009, however, this area has suddenly been 


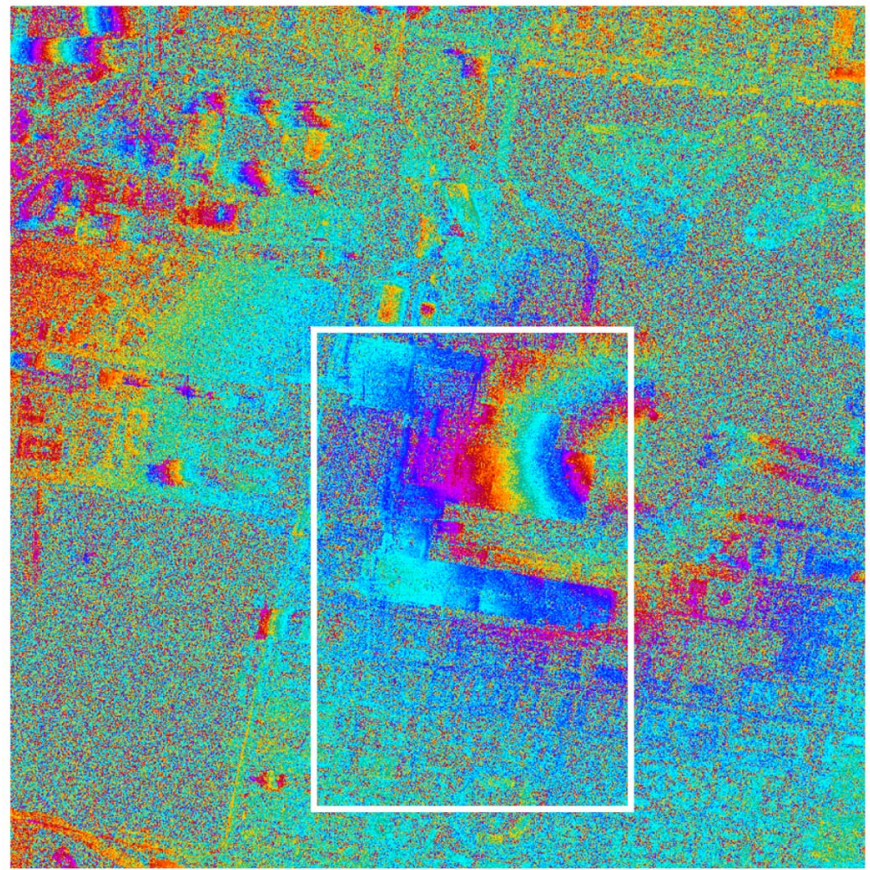

Fig. 6. Differential interferogram of the test site showing a pronounced circular subsidence pattern. The interferogram has been generated by two TS-X images taken from the "diamond" substack in Fig. 5. White box indicates the investigation area in Fig. 9.

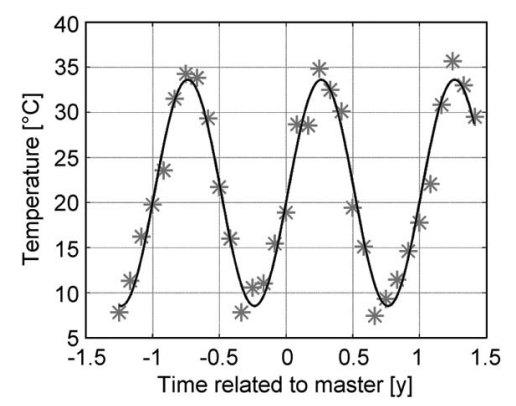

Fig. 7. (Asterisk) Monthly average temperature of Las Vegas, Nevada, in the years of 2008-2010 versus (solid line) modeled seasonal motion with initial offset $t_{0}=0.013$ years.

undergoing a pronounced subsidence centered at the convention center which can be seen from the differential interferogram in Fig. 6 generated by two images taken on April 17, 2009 (master), and April 4, 2010 (slave). Hence, the second-half acquisitions (the "diamond" substack in Fig. 5) are characterized by a multicomponent nonlinear motion, i.e., a combined linear and thermal-dilation-induced seasonal motion. We will use these favorable motion properties to validate the generalized time warp method for single-component and multicomponent nonlinear motion estimation, respectively.

\section{B. Thermal-Dilation-Induced Seasonal Motion Estimation}

In this section, the "asterisk" substack is used to estimate the thermal-dilation-induced motion. Theoretically speaking, we should use the temperature history at the acquisition times as the motion base function and find out the scaling factor. However, due to the lack of ground truth, we use a simple sine function with a period of one year. The initial offset is estimated by fitting the sine model to the monthly average temperature of the test site in the years of 2008-2010 as shown in Fig. 7. The asterisks mark the monthly average temperatures [8], and the solid line drafts the modeled seasonal motion with initial offset $t_{0}=0.013$ years. Likewise, the initial offset can be obtained from the PSI-based preprocessing mentioned in Section IV-A.

The surface model and amplitude map of seasonal motion have been obtained by the proposed time warp method for the whole building complex of the convention center (marked by a box in Fig. 4). Fig. 8 shows the surface model generated from the elevation estimates (converted to height). The shapes of the building and the surrounding infrastructure, e.g., roads and bridges, have been captured at a very detailed level. The right image in Fig. 8 shows the amplitude map of the seasonal motion caused by thermal dilation. Since the building has a metallic structure, it has been more severely affected by the thermal dilation than the surrounding infrastructure. The building shows a smooth amplitude variation for individual structural blocks. There are sudden changes of the seasonal motion amplitude between the adjacent blocks of up to $8 \mathrm{~mm}$. The surrounding area shows almost no seasonal motion.

\section{Linear Motion + Seasonal Motion Estimation}

The generalized time warp method for $M=2$ has been applied to the "diamond" substack in Fig. 5 by choosing linear and seasonal motion as the base functions. The left image in Fig. 9 shows the TS-X intensity map of the region of interest (marked by a box in Fig. 6). According to Fig. 6, the center of the subsidence pattern, i.e., the "epicenter," is located on the right upper part of the intensity map. Therefore, together with the seasonal motion results shown in Fig. 8, we can expect the following: 1) only the building structures suffer from thermal dilation, and 2) the closer to the "epicenter" the point is, the bigger is the linear subsidence.

To validate the proposed generalized time warp method, the two pixels $P_{1}$ and $P_{2}$ marked by red points have been selected and will be analyzed hereinafter (see the left image in Fig. 9; $P$ is the reference point). As $P_{1}$ is located outside the region of the convention center, it is expected to contain only a single-component linear motion. $P_{2}$ is located on the roof and is closer to the "epicenter." Hence, we expect a combined seasonal and more significant linear motion. The middle and right image pairs in Fig. 9 shows the corresponding estimated reflectivity profiles (upper) and scatterer distributions in the $(a-v)$ motion plane (bottom) of the analyzed ground pixel $P_{1}$ and roof pixel $P_{2}$, respectively. The estimates reveal a single scatterer in the pixel $P_{1}$ with an elevation of $-12 \mathrm{~m}(\approx-6.3 \mathrm{~m}$ in height relative to reference pixel $)$, almost no seasonal motion, and a subsidence of $-5 \mathrm{~mm} / \mathrm{year}$. Also, $P_{2}$ is estimated to be a single scatterer, with an elevation of $32 \mathrm{~m}(\approx 16.9 \mathrm{~m}$ in height $)$, a seasonal motion with an absolute amplitude of $4.5 \mathrm{~mm}$, and a subsidence of $-10 \mathrm{~mm} /$ year. These results are consistent with our expectation and, hence, validate the capability of the time warp method for multicomponent nonlinear motion estimation.

\section{CONCLUSion}

The proposed time warp method for D-TomoSAR converts any nonlinear multiple $(M)$ component motion history into a linear one. It renders D-TomoSAR an $(M+1)$-dimensional 

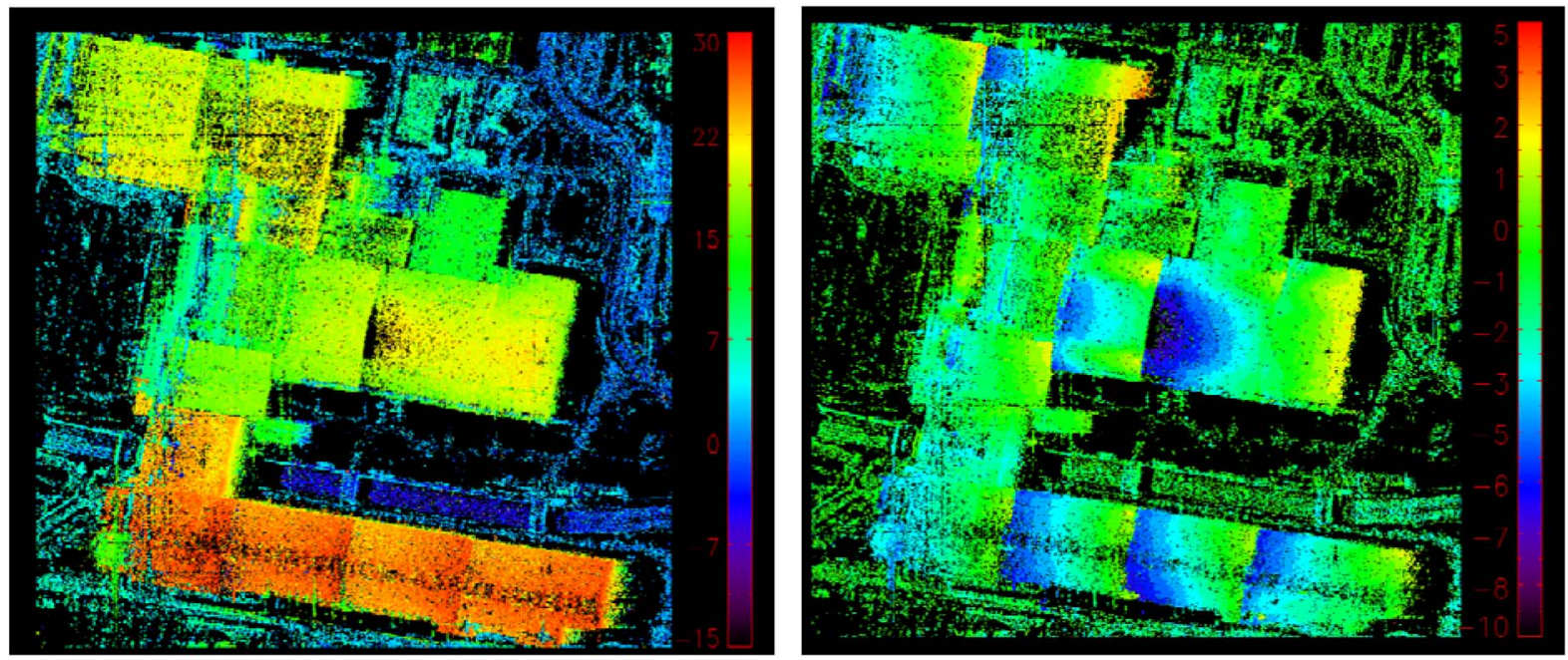

Fig. 8. (Left) Reconstructed digital surface model from differential TomoSAR (unit: meters) and (right) estimated amplitude of seasonal motion (unit: millimeters).
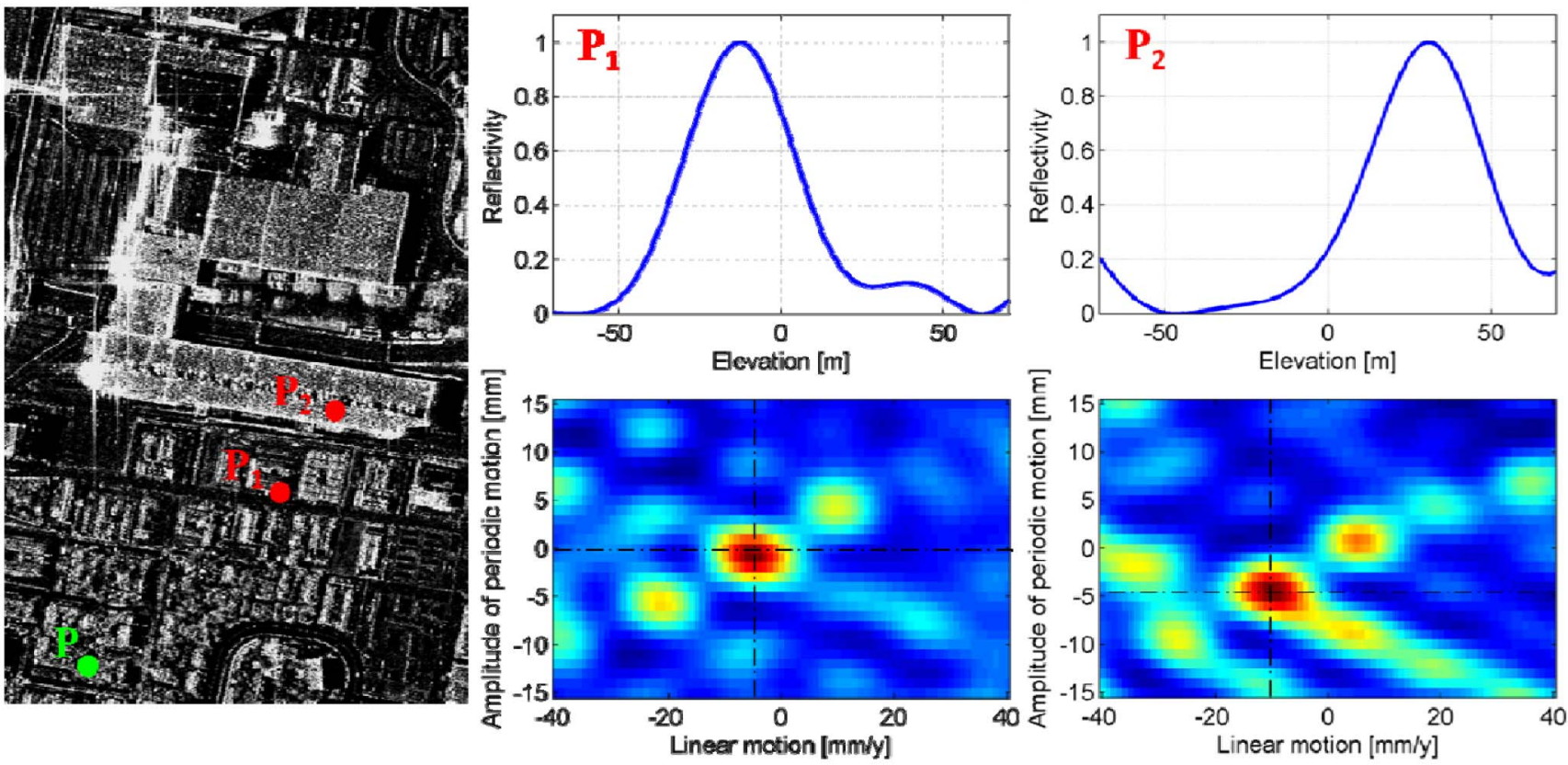

Fig. 9. Validation examples of the generalized time warp approach. (Left) TS-X intensity map. (Middle) (Upper) Reflectivity profile and (bottom) scatterer distribution in $(a-v)$ motion plane of analyzed ground pixel $P_{1}$. (Right) Same plots of analyzed roof pixel $P_{2} . P$ is the reference point. Dashed lines mark the location of the maxima in the spectral estimates.

spectral estimation problem. The choice of the motion base functions reflects our prior knowledge about the underlying motion process. An interesting option is to use GPS measurements in the vicinity of the objects of interest for constructing the base functions. These measurements can provide important features of the motion process, e.g., start time and acceleration rate of a subsidence.

\section{REFERENCES}

[1] F. Lombardini, "Differential tomography: A new framework for SAR interferometry," in Proc. IGARSS, Toulouse, France, 2003, pp. 1206-1208.

[2] G. Fornaro, D. Reale, and F. Serafino, "Four-dimensional SAR imaging for height estimation and monitoring of single and double scatterers," IEEE Trans. Geosci. Remote Sens., vol. 47, no. 1, pp. 224-237, Jan. 2009.
[3] X. Zhu and R. Bamler, "Very high resolution spaceborne SAR tomography in urban environment," IEEE Trans. Geosci. Remote Sens., vol. 48, no. 12 pp. 4296-4308, Dec. 2010.

[4] B. M. Kampes, "Radar interferometry: The persistent scatterer technique," in Remote Sensing and Digital Image Processing Series, 1st ed. New York: Springer-Verlag, 2006.

[5] X. Zhu and R. Bamler, "Tomographic SAR inversion by L1 norm regularization-The compressive sensing approach," IEEE Trans. Geosci. Remote Sens., vol. 48, no. 10, pp. 3839-3846, Oct. 2010.

[6] G. Fornaro, D. Reale, and F. Serafino, "4D SAR focusing: A tool for improved imaging and monitoring of urban areas," in Proc. IEEE Int. Geosci. Remote Sens. Symp., 2008, pp. V-475-V-478.

[7] X. Zhu and R. Bamler, "Very high resolution SAR tomography via compressive sensing," in Proc. ESA FRINGE Workshop, Adv. Sci. Appl. SAR Interferometry, Frascati, Italy, 2009.

[8] Average Daily Temperature Archive. [Online]. Available: http://www.wrh. noaa.gov/vef/motemps.php 